Check for updates

Cite this: Phys. Chem. Chem. Phys., 2018, 20, 20650

Received 19th June 2018 Accepted 24th July 2018

DOI: $10.1039 / c 8 c p 03871 f$

rsc.li/pccp

\section{Large-scale QM/MM free energy simulations of enzyme catalysis reveal the influence of charge transfer $\dagger$}

\begin{abstract}
Heather J. Kulik (iD
Hybrid quantum mechanical-molecular mechanical (QM/MM) simulations provide key insights into enzyme structure-function relationships. Numerous studies have demonstrated that large QM regions are needed to systematically converge ground state, zero temperature properties with electrostatic embedding QM/MM. However, it is not well known if ab initio QM/MM free energy simulations have this same dependence, in part due to the hundreds of thousands of energy evaluations required for free energy estimations that in turn limit QM region size. Here, we leverage recent advances in electronic structure efficiency and accuracy to carry out range-separated hybrid density functional theory free energy simulations in a representative methyltransferase. By studying 200 ps of $a b$ initio QM/MM dynamics for each of five QM regions from minimal (64 atoms) to one-sixth of the protein (544 atoms), we identify critical differences between large and small QM region QM/MM in charge transfer between substrates and active site residues as well as in geometric structure and dynamics that coincide with differences in predicted free energy barriers. Distinct geometric and electronic structure features in the largest QM region indicate that important aspects of enzymatic rate enhancement in methyltransferases are identified with large-scale electronic structure.
\end{abstract}

\section{Introduction}

Mixed quantum mechanical (QM)/molecular mechanical (MM), i.e., $\mathrm{QM} / \mathrm{MM}$ modeling ${ }^{1-9}$ remains the method of choice for simulating enzymes ${ }^{10}$ owing to its ability to balance accuracy in describing chemical rearrangements, polarization, and charge transfer at an enzyme active site with the low computational cost needed to enable sampling. Computational cost in $\mathrm{QM} / \mathrm{MM}$ is typically minimized by employing small QM region sizes on the order of tens of atoms (i.e. ligands and a few direct residues), ${ }^{9,11,12}$ bringing to the fore the study and improvement of QM/MM boundary treatment $\mathrm{t}^{7,13-22}$ and embedding (i.e., polarizable) approach. ${ }^{23-29}$ Nevertheless, QM region size minimization is limited by the inability to describe charge transfer between MM residues and the QM active site. ${ }^{30,31}$ At the same time, advances over the past decade ${ }^{30,32-39}$ in computational efficiency now enable fully $a b$ initio, quantum chemical simulation of polypeptides ${ }^{32,40-46}$ as well as QM/MM treatments of enzymes with large ( $>100$ atoms) $\mathrm{QM}$ regions. These advances have enabled systematic studies ${ }^{47-57}$ that have identified in static calculations a slow approach to asymptotic limits as QM regions are enlarged radially in QM/MM

Department of Chemical Engineering, Massachusetts Institute of Technology, Cambridge, MA 02139, USA. E-mail: hjkulik@mit.edu; Tel: +1-617-253-4584

$\dagger$ Electronic supplementary information (ESI) available. See DOI: 10.1039/c8cp03871f calculations. Such slow approach to asymptotic limit (at ca. 500-1000 atoms) has been observed for wide-ranging properties, including: NMR shieldings, ${ }^{47,48}$ proton transfer, ${ }^{58}$ solvation effects, ${ }^{49}$ barrier heights, ${ }^{50-52}$ forces, ${ }^{53}$ excitation energies, ${ }^{54,59,60}$ partial charges, ${ }^{55}$ bond critical points, ${ }^{61}$ and redox potentials. $^{56}$ This strong sensitivity to QM region size has motivated ongoing method development for general ${ }^{50,62-65}$ or system-specific $^{56}$ QM region determination, including through the response of QM active site residues to perturbation of surrounding environmental residues treated at the $\mathrm{MM}^{50,66}$ or $\mathrm{QM}^{52,67,68}$ level, to enable atom-economical, quantitative QM/MM.

Although electronic structure calculations on over 1000 atoms have become increasingly routine, a potential of mean force requires hundreds of thousands of energy evaluations, meaning that large-QM (i.e., >100 atoms) QM/MM free energy calculations have overwhelmingly been carried out ${ }^{53,69-73}$ with semi-empirical methods that depend strongly on the quality of parameterization ${ }^{74}$ for adequate description of long-range charge transfer, hydrogen bonding, and electrostatic interactions. In order to assess (i) whether qualitative differences emerge from large-scale $\mathrm{QM} / \mathrm{MM}$ and (ii) how sensitive QM/MM free energy simulations (FES) are to QM region size, we carried out the first large-scale, range-corrected, hybrid density functional theory (DFT) QM/MM free energy simulations with very large (>500 atom) QM regions. For this study, we select catechol $O$-methyltransferase (COMT), 
a representative member of a large class of $S$-adenosyl-Lmethionine (SAM)-dependent ${ }^{75}$ methyltransferases (MTases) responsible for gene signaling, ${ }^{76}$ protein repair, ${ }^{77,78}$ and neurotransmitter regulation. ${ }^{75}$

The outline of the rest of this work is as follows. In Section 2, we review the computational details of the QM/MM free energy simulations. In Section 3, we present the reasoning for selected QM regions in QM/MM FES simulations of COMT and subsequent observations of energetic, structural, and electronic properties for differing QM regions. Finally, in Section 4, we present our conclusions.

\section{Computational details}

QM/MM dynamics with electrostatic embedding and hydrogen link atoms, i.e.:

$$
E_{\mathrm{QM} / \mathrm{MM}}=E_{\mathrm{QM}}+E_{\mathrm{QM} / \mathrm{MM}}+E_{\mathrm{MM}}
$$

employed the $\mathrm{AMBER}^{79,80}$ interface $^{54}$ to TeraChem. ${ }^{35,81} \mathrm{QM}$ atoms were treated at the $\omega \mathrm{PBEh}^{82} / 6-31 \mathrm{G}^{* 83}$ level of theory with semi-empirical (i.e., D3 ${ }^{84}$ ) dispersion, and the $\mathrm{MM}$ atoms were treated with the ff14SB AMBER force field ${ }^{85,86}$ or TIP3P ${ }^{87}$ for water, as motivated by tests in previous work. ${ }^{52,67}$ Specifically, for numerous snapshots, it was observed that the $6-31 \mathrm{G}^{*}$ basis produced consistent results with those obtained after incorporation of diffuse functions and additional polarization functions. ${ }^{67}$ A range-separated hybrid was chosen after it was observed that some electronic properties, including spatial placement and energetic gap of the frontier orbitals, were erroneous with global hybrids. ${ }^{52,67}$ These dynamics were carried out with spherical boundary conditions in a 41 A radius sphere extracted from an NpT-equilibrated rectangular boundary conditions box from ref. 70. The sphere was simulated with no electrostatic cutoff and a $1.5 \mathrm{kcal} \mathrm{mol}^{-1} \AA^{-2}$ restraining potential at the boundary (see ESI $\dagger$ ). A 0.5 fs timestep was used and constant temperature $(T=300 \mathrm{~K})$ was enforced by a Langevin thermostat. These 27852 atom starting structures were obtained from prior $^{70}$ classical MD and semi-empirical QM/MM (SQM/MM) equilibration of COMT (PDB ID: $3 \mathrm{BWM},{ }^{88}$ protonation states given in ESI, $\dagger$ Table S1). In that prior work, ${ }^{70}$ the SQM region contained substrates, $\mathrm{Mg}^{2+}$ and its coordinating residues (D141, D169, N170, water), along with nearby E199 and K144 residues, whereas the remaining atoms were in the MM region. This -1 net charge, 147 atom SQM region is similar to the M2 region in the present work (see Section 3).

The reaction coordinate in umbrella sampling was defined as an antisymmetric linear combination of distances (LCOD) between the $\mathrm{S}-\mathrm{C}$ and $\mathrm{C}-\mathrm{O}$ bonds, which break and form, respectively, during methyl transfer. Variable force constants ranging from 10 to $240 \mathrm{kcal} \mathrm{mol}{ }^{-1} \AA^{-2}$ were employed to minimize the number of windows required, which was 14 in total, while maximizing overlap over the LCOD range (details provided in ESI, $\uparrow$ Table S2). The weighted-histogram analysis method (WHAM), ${ }^{89,90}$ as implemented in the Grossfield lab software package, ${ }^{91}$ was used to reconstruct one-dimensional

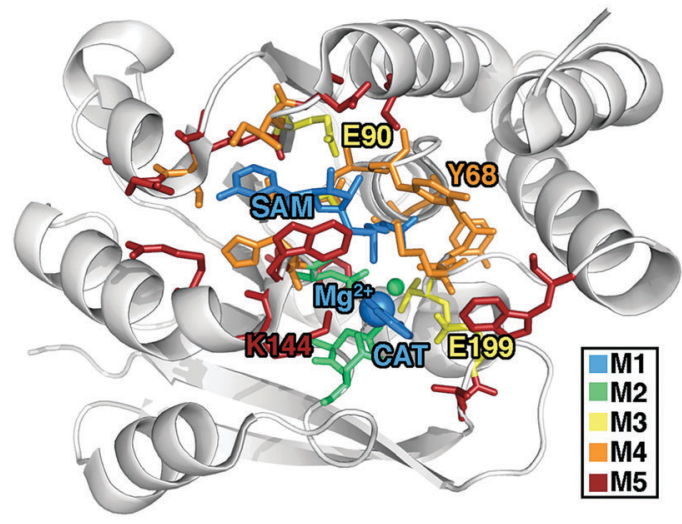

Fig. 1 COMT structure with residues included in 5 QM region models shown as sticks, colored according to the smallest model in which they first appear: SAM, $\mathrm{Mg}^{2+}$, and CAT substrates-only model $(64$ atom, +2 net charge M1, blue), $\mathrm{Mg}^{2+}$ coordination sphere (109 atom, 0 net charge M2, green), critical residues (170 atom, -2 net charge $\mathbf{M 3}$, yellow), full systematic QM/MM region (325 atom, -3 net charge M4, orange), and a large QM model (544 atom, -1 net charge M5, red).

free energy curves with $0.02 \AA$ bin widths from umbrella sampling. ${ }^{92}$ Starting from the endpoint of SQM/MM dynamics (30 ps per window in ref. 70), a 2 ps equilibration at the QM/ MM level was discarded before completing 15 ps production runs for the free energy calculations in five QM/MM regions ranging from 64 atoms to 544 atoms in size for a total of $1.05 \mathrm{~ns}$ production QM/MM dynamics (Fig. 1 and ESI, $\dagger$ Table S3). Mulliken partial charges, computed at each time step, were analyzed by summing over each residue to minimize errors in partial charge assignment intrinsic in the Mulliken approach. The methyl partial charge is partitioned between SAM and CAT according to progress along the reaction coordinate, as in previous work. ${ }^{52}$

\section{Results and discussion}

We investigate the effects of QM region size on dynamics and free energy evaluations in catechol $O$-methyltransferase (COMT), a representative member of a large class of methyltransferases (MTases). ${ }^{75}$ COMT is an $S$-adenosyl-L-methionine (SAM)- and $\mathrm{Mg}^{2+}$-dependent MTase ${ }^{75}$ that reacts with catecholamine substrates bound in a bidentate fashion to $\mathrm{Mg}^{2+}$ at the active site ${ }^{93}$ (Fig. 1). The rate-determining, ${ }^{94}$ direct $\mathrm{S}_{\mathrm{N}} 2$ methyl transfer ${ }^{95}$ from SAM $^{96}$ to a Lys-deprotonated catecholate ${ }^{97,98}$ (CAT) has been the focus of kinetic studies ${ }^{93,99-101}$ that estimate a free energy barrier of $18.1^{100}-19.2^{101} \mathrm{kcal} \mathrm{mol}^{-1}$ for the soluble, human form of the enzyme. COMT has also been the focus of numerous DFT ${ }^{52,102,103}$ and semi-empirical ${ }^{70-72,104,105}$ computational studies ${ }^{71,102-106}$ that have produced a wide range (i.e., 3-30 kcal mol${ }^{-1}$ ) of free energy barriers that can be brought into agreement with experiment through various corrections (e.g., a spline that approximates differences between semiempirical and MP2 energies ${ }^{107}$ ).

We previously identified that static $\operatorname{properties}^{52}$ of COMT (e.g., barrier heights, and structures) obtained with range-separated 
hybrid DFT are slow to converge to asymptotic large-QM limits ( $c a$. 300-500 atoms) through an evaluation of these properties with 10 increasing radial cutoffs for protein residue and water inclusion in the QM region. We then developed systematic methods to identify which residues were most essential in static calculations. ${ }^{52,67,68}$ Using these methods, ${ }^{52,67,68}$ we obtained $0 \mathrm{~K} \mathrm{QM} / \mathrm{MM}$ activation energies and reaction energies within $1 \mathrm{kcal} \mathrm{mol}^{-1}$ of the asymptotically converged result with around half the number of atoms in the radially converged region (around 200 vs. 500 atoms). The systematic selection methods focused on charge transfer between residues to reveal essential hydrogen bonding interactions and salt bridges as well as surprising nonpolar contributions to the enzyme active site. ${ }^{67}$ If interactions were captured across the $\mathrm{QM} / \mathrm{MM}$ boundary, despite proximity (e.g., a lysine proximal to a glutamate near the active site), then those residues were not selected for the QM region. Now, we investigate if the regions that were shown to be asymptotically converged in $0 \mathrm{~K} \mathrm{QM} / \mathrm{MM}$ show similar or even reduced QM region size dependence than the prior $0 \mathrm{~K}$ studies.

For the largest-scale QM/MM free energy simulations in this work, we select a QM region of 544 atoms (28 protein residues) motivated by these prior ${ }^{52,67}$ convergence studies and employ range separated hybrid DFT in a polarized double- $\zeta$ basis set. Both basis and method are selected as a compromise for what is presently feasible to carry out the $>400 \mathrm{k}$ energy evaluations on the largest QM region selected (see Computational details and ESI, $\uparrow$ Table S3). Our smaller QM regions are motivated by prior studies or our own systematic QM region construction results for convergence of static properties: ${ }^{67}$ (i) M1 (64 atoms, +1 net charge): CAT, SAM, and $\mathrm{Mg}^{2+}$ only, (ii) M2 (109 atoms, 0 net charge): added $\mathrm{Mg}^{2+}$ coordination sphere (D141, D169, and N170), (iii) M3 (170 atoms, -2 net charge): four additional key residues, V42, G66, E90, and E199, from static substratedeletion analysis, (iv) M4 (325 atoms, -3 net charge): nine more residues from systematically-selected QM regions for static calculations, ${ }^{67}$ and (v) M5 (544 atoms, -1 net charge): a 28-residue region obtained from prior radial convergence studies ${ }^{52}$ (see Fig. 1 and ESI, $\dagger$ Table S3). The system sizes span an order of magnitude from 64 atoms with no link atoms for M1 to 544 atoms including 26 link atoms for M5 (see Computational details). Because each free energy simulation requires nearly half a million single point energy evaluations, we took inspiration from our prior studies to select QM regions ranging from minimal in size (M1 or M2) to systematically converged (M4 and M5) or nearly converged (M3) at the $0 \mathrm{~K} \mathrm{QM} / \mathrm{MM}$ limit observed for several 600-1000 atom QM regions. ${ }^{52,67}$ Thus, the central theme of this work is to identify if free energy simulations have similar or reduced sensitivity to QM region size as our prior $0 \mathrm{~K} \mathrm{QM} / \mathrm{MM}$ simulations. ${ }^{52,67}$ In the present work, the single water within $4 \AA$ of the reacting atoms on SAM and catecholate is included in the QM region, motivated by (i) the fact that more distant ( $c a$. 5-6 ̊) water molecules were shown to have limited effect on $0 \mathrm{~K}$ barrier heights in prior work $^{52}$ and (ii) that our fixed QM boundary approach would not be equipped to handle water diffusion across the boundary during the dynamics of the free energy simulations.

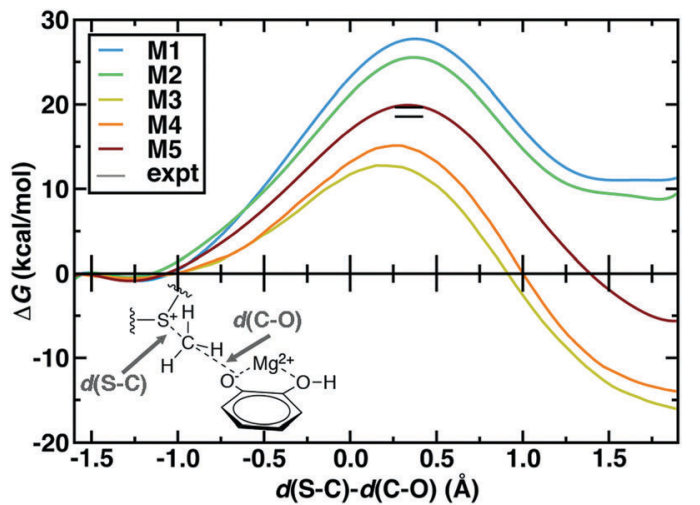

Fig. 2 Potentials of mean force $\left(\mathrm{kcal} \mathrm{mol}^{-1}\right)$ for $\mathrm{QM}$ regions $\mathrm{M} 1$ through M5 plotted against the LCOD as defined in the main text and shown in bottom left inset. The experimental range ${ }^{100,101}$ is indicated by horizontal lines.

The 544 atom model (M5) predicts a $\Delta G^{\ddagger}$ of $19.9 \mathrm{kcal} \mathrm{mol}^{-1}$ that is within 1-2 $\mathrm{kcal} \mathrm{mol}^{-1}$ of best experimental estimates ${ }^{100,101}$ (Fig. 2). However, similar agreement with experimental $\Delta G^{\ddagger}$ has been achieved with a number of levels of theory and QM region sizes in prior work. Using a combination of long-time classical and semi-empirical (i.e., PM6 ${ }^{108}$ ) SQM/MM dynamics, we previously determined ${ }^{70}$ that semi-empirical barrier heights were sensitive to inclusion of the $\mathrm{Mg}^{2+}$ coordination sphere and residues adjacent to the substrates. The inclusion of both types of residues produced barrier heights (ca. 21-22 $\mathrm{kcal} \mathrm{mol}^{-1}$ ) in nearly as good agreement with experiment as the present largeQM/MM simulations. ${ }^{109}$ Concurrent with our PM6 SQM/MM COMT study, ${ }^{70}$ a complementary SQM/MM study ${ }^{72}$ was published in which 15 PM6 SQM regions were compared to study methyl transfer between a partial model of SAM (i.e., $\left.\mathrm{S}\left(\mathrm{CH}_{3}\right)_{3}{ }^{+}\right)$ and catecholate in COMT. In both studies, ${ }^{70,72}$ the importance of the inclusion of $\mathrm{Mg}^{2+}$ and its coordination sphere in the SQM region were recognized. Therefore, in 13 of the models studied in ref. $72, \mathrm{Mg}^{2+}$, sidechain-only models of the $\mathrm{Mg}^{2+}$ coordination sphere, and the sidechains of up to four additional protein residues were included in the SQM region. These 13 sub-100 atom SQM regions produced variations of the barrier height of approximately $5 \mathrm{kcal} \mathrm{mol}^{-1},{ }^{72}$ with all models underestimating the experimental barrier height, as would be expected from the use of SQM/MM methods. Larger variations $\left(\sim 20 \mathrm{kcal} \mathrm{mol}^{-1}\right)$ were seen in the free energy of reaction. ${ }^{72}$ Both SQM/MM studies $^{70,72}$ highlighted that reaction free energies were in fact even more sensitive to QM region size than the free energy barrier, consistent with expectations from Brønsted-EvansPolanyi relations. However, they also seemed to indicate that relatively small SQM regions could be chosen to achieve reasonable agreement (i.e., within $5 \mathrm{kcal} \mathrm{mol}^{-1}$ ) with the experimental $\Delta G^{\ddagger} .^{70,72}$ Our present work differs from these prior studies because, despite these apparent successes, the reliability of SQM to predict essential energetic ${ }^{110}$ or density ${ }^{111}$ properties governed by an appropriate balance of non-covalent interactions, long-range charge transfer, and electrostatics remains an active area of research. ${ }^{74}$ Although corrections have been 
developed to address potential limitations, they are achieved by fitting to higher levels of theory on small gas phase test sets. ${ }^{112}$ The present work directly treats a full model of SAM and a much larger portion of the protein environment with rangeseparated hybrid DFT, which improves treatment of long-range charge transfer over both SQM methods and DFT with global hybrids or semi-local exchange-correlation functionals. ${ }^{40,52,113}$

First, rather than aiming for agreement of the theoretical and experimental free energy barriers, which might be arrived at without a complete description of the active site chemistry, we focus on electronic structure properties and dynamics at the active site of our large-scale QM/MM simulation. We will then return to the question of understanding the degree of holistic consistency of energetic, geometric, and electronic structure properties of the five QM regions we have studied. Based on gas phase models, it has been argued ${ }^{106}$ that the positively charged SAM and negatively charged CAT become neutral species at the transition state (TS), eliminating the electrostatic driving force for the reaction. The role of the greater enzyme environment in COMT has been debated, ${ }^{52,71,101,114}$ but the unexpectedly short non-bonded SAM-CAT distance observed experimentally ${ }^{88}$ and in $\mathrm{QM} / \mathrm{MM}$ geometry optimizations ${ }^{52,114}$ appears electrostatically driven. Indeed, in our dynamics, we observe charge transfer mediated prolonged electrostatic attraction across the reaction coordinate defined by the difference in bond length of the cleaving S-C and forming $\mathrm{C}-\mathrm{O}$ bonds (Fig. 3a). At the TS, the summed Mulliken partial charges on SAM (positive) and CAT (negative) fragments are roughly $2 / 3$ of the magnitude they are in the Michaelis complex $(d(\mathrm{~S}-\mathrm{C})-d(\mathrm{C}-\mathrm{O}), \Delta \sim-1 \AA$, see Fig. 3$)$. Only when the product is fully formed do the SAM- and CAT-summed charges approach zero, even when taking into account dynamic fluctuations in partial charges on both substrates (shaded ranges in Fig. 3a). It is also noteworthy that the SAM substrate reaches a peak positive formal charge near the shortened non-bonded distances observed in crystal structures between the substrates (ca. $\Delta=-0.8 \AA, d(\mathrm{~S}-\mathrm{C})=1.82 \AA d(\mathrm{C}-\mathrm{O})=2.62 \AA)$ likely due to proximity and enhanced charge transfer to N41/V42 from the SAM carboxylate (ESI, $\dagger$ Fig. S2 and Tables S4-S6).

The net charge on the combined positive SAM and negative CAT substrates represents the degree of charge transfer between substrates and the surrounding enzyme environment. If this quantity remains close to zero in the QM/MM free energy simulations, then we can expect that increasingly accurate embedding $^{23-29}$ (i.e., polarizable) might be able to capture prolonged electrostatic attraction between residues. However, we observe a wide distribution of net charge from -0.4 to 0.4 on the substrates during our simulation, and only $1 / 3$ of the simulation time is spent with the summed substrate charge within $\pm 0.05 e$ (Fig. 3b). Decomposing the formal charge across the reaction coordinate reveals that a formal positive charge (charge transfer away from the substrates) mediates the shortened Michaelis complex structures observed earlier, whereas charge accumulates on the substrates leading up to the TS and in the product (Fig. $3 \mathrm{~b}$ and ESI, $\dagger$ Fig. S3 and S4).

One might expect that the charge transfer between the substrates and the surrounding environment could be localized
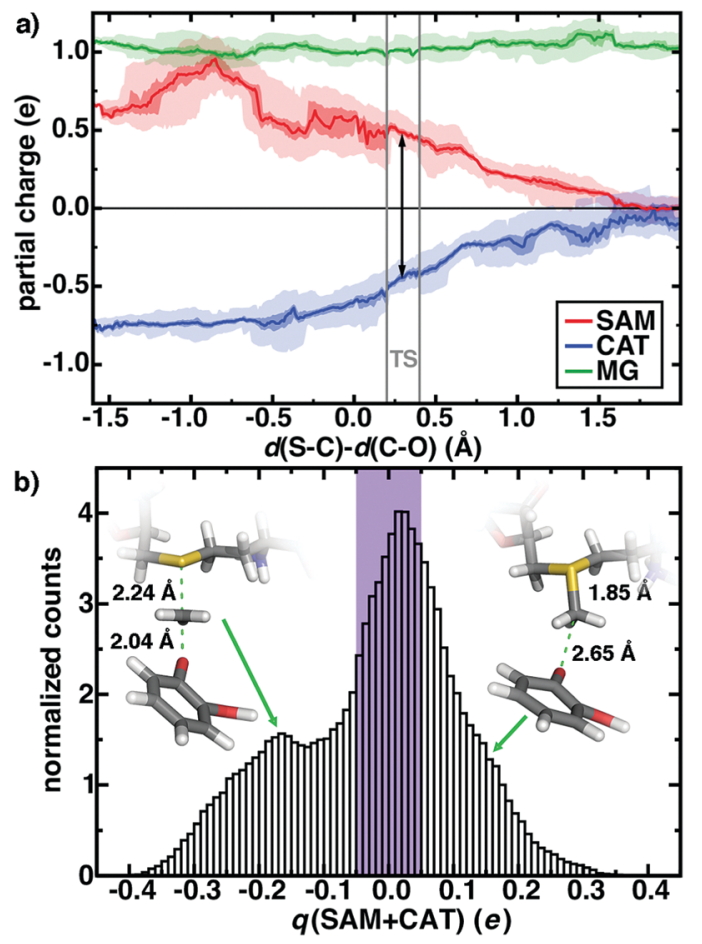

Fig. 3 (a) Sum of charges on SAM (red), CAT (blue), and $\mathrm{Mg}^{2+}$ (green) during methyl transfer reaction in the M5 QM/MM model. Over $0.01 \AA$ bins of simulation data, average charges are indicated by lines, the first to third interquartile range is indicated by the darker shaded region and the full range of charges are indicated by light shading. The methyl charge is partitioned between substrates as described in the main text, and the TS region is indicated as gray vertical bars with average charge separation shown as a black arrow. (b) Normalized histogram of partial charges summed over SAM and CAT (in e) across 200 ps of aggregated M5 free energy simulation. The purple bars represent \pm 0.05 e range and each bin is 0.01 e wide.

to only a few charged residues within hydrogen bonding distance of the substrates. We computed by-residue partial charges and compared the dependence of maximum fluctuations (i.e., the difference of maximum and minimum by-residue charges observed during the simulation) to the closest heavy atom distance between the residue and $\mathrm{SAM} / \mathrm{CAT} / \mathrm{Mg}^{2+}$ substrates averaged over the reactant, TS, and product geometries (Fig. 4 and ESI, $\dagger$ Table S4). We observe very large charge fluctuations (ca. 0.7 e) for some proximal and charged residues (e.g., E199) but moderate fluctuations for all residues in the QM region of this model (min. of $0.08 e$ ) and surprisingly little closest-heavyatom-distance dependence (ESI, $\uparrow$ Fig. S2 and Tables S4-S6). To test if charge fluctuations are still localized between pairs of hydrogen bonding residues, we also summed the partial charges of the substrates and the 14 residues with closest heavy atom distances within 3.0 ̊ of the substrates. This portion of the M5 QM region exhibits just as large fluctuations in deviations from formal charge, with at least three distinct peak features in a wide -0.5 to $+0.2 e$ deviation range and only $26 \%$ of all frames within $0.05 e$ of the expected formal charge (ESI, † Fig. S5).

As an example of weak distance dependence, D141 and D169, which form the $\mathrm{Mg}^{2+}$ coordination sphere, exhibit lower 


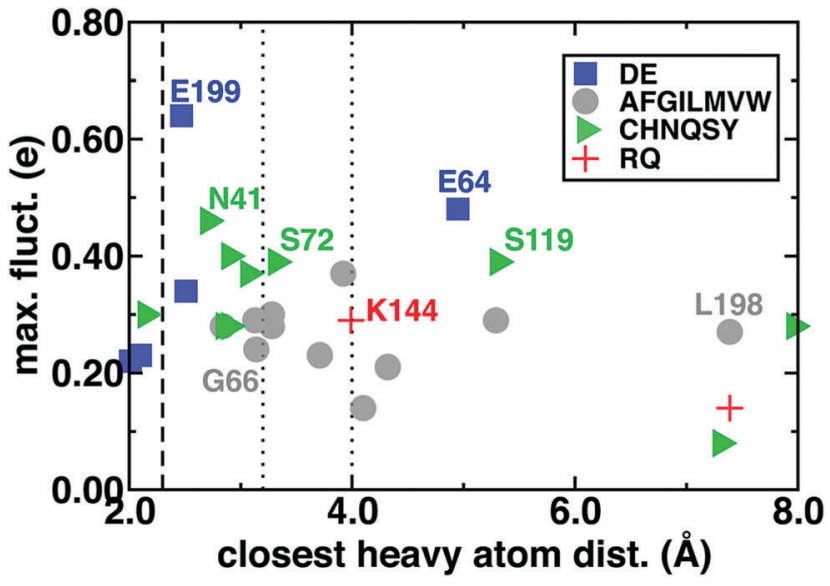

Fig. 4 Range of by-residue summed partial charges including link atoms (max. fluct., e) sampled for each residue grouped by negatively charged (blue squares), nonpolar (gray circles), polar (green triangles), and positively charged (red crosses), with residue types indicated in legend plotted against the average closest heavy atom distance between the residue and SAM, CAT, or $\mathrm{Mg}^{2+}$. Vertical lines indicate approximate covalent bond (dashed), moderate hydrogen bond (dotted), and weak hydrogen bond (dotted) distance cutoffs.

fluctuations than nonpolar L198, which is $7 \AA$ from the closest substrate heavy atoms (Fig. 4). Perhaps surprisingly, similar magnitude fluctuations are observed for both polar and nonpolar residues, and a comparison of charge standard deviations preserves trends (ESI, $\uparrow$ Fig. S6-S8). Large fluctuations in residues beyond typical hydrogen bonding distances to the substrates (i.e., $\sim 3.25 \AA$, see Fig. 4) can be rationalized by proximity to substrate-neighboring residues that have large fluctuations. For example, the relatively high fluctuations for substrate-distantce L198 are a consequence of its being adjacent to E199, and the somewhat distant E64 hydrogen bonds with S72, which in turn hydrogen bonds with SAM (see ESI $\dagger$ ). On the other hand, D141 and D169 have among the lowest fluctuations for charged residues, despite significant deviations from formal charges (ESI, $\dagger$ Fig. S9). This highlights that fluctuations arise predominantly in mobile residues that vary in active site proximity along the reaction coordinate rather than protein residues that remain covalently coordinated to the substrates (ESI, $\dagger$ Table S4).

The delicate interplay of charge transfer between the substrate and the enzyme environment can be observed through the structure of the reacting substrates and $\mathrm{Mg}^{2+}$, also highlighting the crucial importance of $\mathrm{Mg}^{2+}$ in COMT catalysis. ${ }^{75}$ The four $\mathrm{Mg}^{2+}-\mathrm{O}$ bonds with the protein, i.e., D141, D169, $\mathrm{N} 170$, and a water molecule are relatively unchanged across the reaction coordinate but differ in length (Fig. 5). Although D169 and D141 $\mathrm{Mg}^{2+}-\mathrm{O}^{-}$bonds are shorter than the neutral N170 bond (2.02-2.10 $⿱$ \& vs. $2.17 \AA$ ) as expected, the $\mathrm{Mg}^{2+}$-water bond is substantially shorter $(2.02 \AA)$ than observed for hexa-aqua complexes $^{115}$ (Fig. 5 and ESI, $\dagger$ Text S1). Across the reaction coordinate, charge transfer mediated prolonged electrostatic attraction is evident in the $\mathrm{Mg}^{2+}-\mathrm{O}$ bond of the catecholate oxygen that carries out nucleophilic attack on the SAM methyl group.

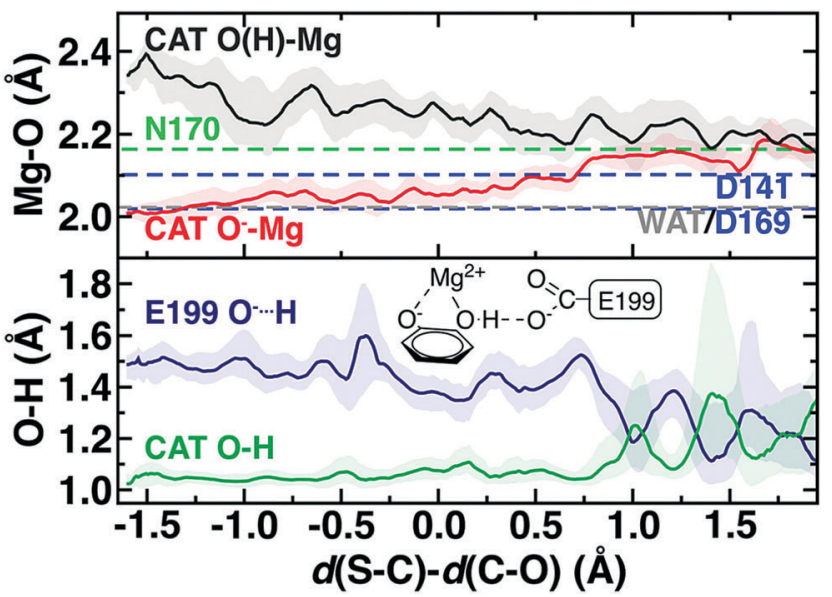

Fig. $5 \mathrm{Mg}^{2+}$ coordination sphere (top) and catecholate (bottom) structural properties in the M5 QM/MM model across the reaction coordinate. At top, the median, averaged distance is reported as dashed lines for N170 (green), D141 and D169 (blue), and water (WAT, gray) as a reference for the CAT $\mathrm{O}(\mathrm{H})$ (dark gray) and CAT $\mathrm{O}^{-}$(red) distances to $\mathrm{Mg}^{2+}$ across the reaction coordinate. At bottom, catechol hydrogen atom distance to neighboring E199 (dark blue) and to the catechol oxygen atom (dark green) are plotted along the reaction coordinate. The remaining quantities are the median values obtained at $0.01 \AA$ increments and averaged over a $0.1 \AA$ window with the range of variations shown as a shaded region. A schematic of E199, $\mathrm{Mg}^{2+}$, and CAT is shown in inset at bottom.

Initially, this bond is the shortest ( $c a .2 .00 \AA$ ) of all $\mathrm{Mg}^{2+}-\mathrm{O}$ bonds and remains in the range of the D141 and D169 $\mathrm{Mg}^{2+}$-carboxylate bond lengths until well past the TS (ca. $\Delta=0.7 \AA, d(\mathrm{~S}-\mathrm{C})=2.59 \AA d(\mathrm{C}-\mathrm{O})=1.89 \AA)$. Thus, $\mathrm{Mg}^{2+}$ plays the critical role not only of positioning catechol for nucleophilic attack but in maintaining the strong nucleophilic character of the catecholate oxygen. This observation is consistent with prior work that revealed ${ }^{70}$ the importance of a QM description of $\mathrm{Mg}^{2+}$ to capture this interaction to reproduce experimental structural observations, which was frequently neglected in prior SQM/MM studies. ${ }^{107}$

In comparison, the catechol oxygen distance to $\mathrm{Mg}^{2+}$ is the longest in the octahedral coordination sphere, becoming comparable in length to the methylated oxygen- $\mathrm{Mg}^{2+}$ distance only in the product (Fig. 5). The long catechol- $\mathrm{Mg}^{2+}$ bond length is likely due to competition with a strong hydrogen bond that forms with the E199 carboxylate, leading to a shared hydrogen atom between the two species in the product state (both $\mathrm{O}-\mathrm{H}$ ca. $1.2 \AA$ A) typically indicative of a low-barrier hydrogen bond ${ }^{116}$ that would be unlikely to be suitably described across a QM/MM boundary (Fig. 5). Thus, it would appear that charge transfer effects only observable with large QM regions can influence both energetics and structure/dynamics across the reaction coordinate.

The remaining question is to what extent these large-scale $\mathrm{QM} / \mathrm{MM}$ observations of geometric and electronic structure trends are preserved in smaller models in spite of moderate discrepancies in energetics (Fig. 2). In order to evaluate QM region dependence in dynamics, we now compare our four increasingly large QM regions (M1-M4) to our largest QM/MM simulation (M5). Wide ranges of both $\Delta G^{\ddagger}$ and $\Delta G_{\mathrm{r}}$ as well as TS character are 


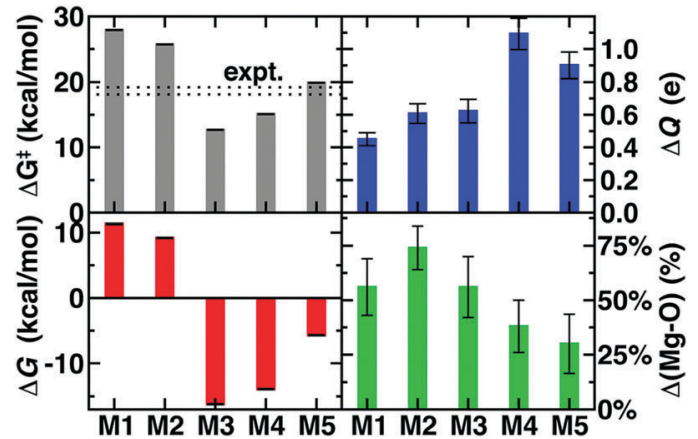

Fig. 6 Comparison of $\Delta G^{\ddagger}$ (in kcal $\mathrm{mol}^{-1}$ ), $\Delta G_{\mathrm{r}}$ (in kcal mol${ }^{-1}$ ), charge separation of SAM and CAT at the TS $(\Delta Q$ in e), and anionic catecholate$\mathrm{Mg}^{2+}$ bond length progress in the TS with respect to difference between the reactant and product (in \%) for five QM regions. Error bars for top and bottom left hand side panels come from bootstrapping and they correspond to standard deviations in quantities on the top and bottom right.

observed across M1-M5 (Fig. 2 and 6 and ESI, $\dagger$ Table S7). Consistent with prior SQM/MM $\mathrm{M}^{70,72}$ and trends in our static results, ${ }^{52}$ both $\mathbf{M} 1$ and $\mathbf{M} 2$ substantially overestimate the $\Delta G^{\ddagger}$, predict an uphill $\Delta G_{\mathrm{r}}$, and underestimate the charge separation at the transition state with respect to M5 (Fig. 6 and ESI, $\dagger$ Table S7). The 170 atom $\mathbf{M} 3$ region incorporates four proximal residues beyond those in the $\mathrm{Mg}^{2+}$ coordination sphere, overstabilizing the transition state and product (i.e., $\Delta G^{\ddagger}(\mathbf{M} 3)<$ $\Delta G^{\ddagger}$ (M5) or expt.), despite underestimating the separation of the substrate charges in the transition state and predicting substrate neutralization in advance of the products (Fig. 6 and ESI, $\dagger$ Fig. S10). Analysis of partial charges reveals that the absence of N41/V42 interactions with the SAM carboxylate likely accounts for the more neutral overall SAM charge but an absence of charge transfer with a QM K144 adjacent to the catecholate overestimates the nucleophilic character of the attacking catecholate oxygen.

Finally, M4 was selected systematically ${ }^{52,67,68}$ on the basis of charge/Fukui shift analysis on $200 \mathrm{~K}$ snapshots of COMT and confirmed to have a $0 \mathrm{~K}$ barrier height within $1 \mathrm{kcal} \mathrm{mol}^{-1}$ of $0 \mathrm{~K}$ converged (i.e., M5) values. ${ }^{67}$ Agreement of QM/MM dynamics, energetics, and charge density between M4 and M5 is slightly worse than the equivalent regions in prior study ${ }^{67}$ but by far in the best agreement of any two regions (Fig. 6). Like M3, M4 overstabilizes the TS and products with respect to M5 but does so by overestimating the charge separation at the TS, suggesting enhanced electrostatic attraction as the driving force for M4 barrier underestimation (Fig. 6). The differences in characteristics between M4 and M5 can likely be attributed to the absence of K144 from the M4 QM region, which was distant from the substrate in the selected $0 \mathrm{~K}$ snapshots but proximal throughout the $300 \mathrm{~K}$ dynamics in this work (ESI, $\dagger$ Table S4). The inclusion of K144 in the M5 QM region has the additional effect of producing a shorter $\mathrm{Mg}^{2+}-\mathrm{O}^{-}$CAT bond in the TS for M5 than in the other 4 QM regions (Fig. 6 and ESI, $\dagger$ Table S8). Overall, the static-QM-selected regions M3 and M4 bear the best qualitative agreement with the large-scale M5 region. Overall, we find greater disagreement (i.e., 3-5 kcal mol${ }^{-1}$ free energy differences $v s$. $0-1 \mathrm{kcal} \mathrm{mol}^{-1} 0 \mathrm{~K}$ electronic energy differences) among QM regions that had been previously systematically converged for $0 \mathrm{~K}$ properties, ${ }^{67}$ emphasizing that deviations are likely due to enhanced sensitivity during dynamics. This observation over QM regions that differ by as much as 375 atoms in size is consistent with prior observations made of $5 \mathrm{kcal} \mathrm{mol}^{-1}$ free energy barrier fluctuations for varying SQM regions of much more similar ( $c a .100$ atoms) size. ${ }^{72}$ Remaining differences highlight that quantitative agreement through systematic QM region selection will require sampling $^{117}$ a wider range of possible configurations relevant during dynamics. Furthermore, as computing power continues to increase, even larger QM regions and longer timescales in $\mathrm{QM} / \mathrm{MM}$ dynamics should be employed to identify the limits of convergence beyond this study.

\section{Conclusions}

In conclusion, we have leveraged accelerated electronic structure approaches to carry out a large-scale study of over $1 \mathrm{~ns}$ of $a b$ initio, range-separated hybrid DFT QM/MM dynamics with QM regions from 64 to 544 atoms in size. These largescale QM/MM studies revealed QM-region-dependent differences in electronic and geometric structure. In addition to accurate prediction of the free energy barrier for methyl transfer, charge separation and neutralization only in the product structure are observed with large QM/MM free energy simulations. Differences in geometry (e.g., relative $\mathrm{Mg}^{2+}$-catecholate oxygen bond lengths) and $>0.4 e$ fluctuations of summed charge on residues $5 \AA$ away from the substrates (i.e., E64 or S119) indicate the extent to which substrate-active site charge transfer can play a key role in enzyme mechanism. This long-range substrate-active site charge transfer is consistent with notions that the enzyme creates an electrostatic driving force for the reaction by stabilizing the transition state but highlights that in some cases large QM regions may be needed to fully observe this effect. These studies also reveal that differences in electronic environments give rise to differences in dynamics and structure in a manner that will likely enhance not decrease QM region dependence for free energy simulations and dynamics. Such observations should motivate the continued improvement of QM/MM through (i) improved embedding schemes and boundary treatment, including to allow charge transfer, (ii) incorporation of large, beyond-DFT QM regions (e.g., through reduced-scaling correlated wavefunction theory), and (iii) development of efficient sampling techniques and free energy decomposition. We note, however, that the large number of hydrogen bonds between charged residues and substrates in the active site of COMT and homologous MTases likely increase QM region dependence over a number of cases where small QM-region QM/MM studies may still encapsulate essential physics.

Although the best agreement with the largest QM region was obtained from systematically constructed QM/MM regions (325 atoms vs. 544 atoms in size) obtained from analysis of two dozen 0 K snapshots, variations between the M4 and M5 QM 
regions in energetics and properties were still apparent. These differences were observed to be due to fluctuations in the orientations of residues during dynamics absent from the $0 \mathrm{~K}$ geometry optimizations and barrier evaluations in previous work that had shown smoother convergence with QM region size. As accelerated electronic structure calculations and computing power become increasingly available, it is anticipated that these calculations could be repeated with alternative QM regions of even larger size to confirm stricter property convergence (e.g., free energies within $1 \mathrm{kcal} \mathrm{mol}^{-1}$ ). Overall, this work also reinforces the importance of systematic QM region construction including dynamic benchmarking of substrate electronic properties to identify minimal sufficient QM regions in future QM/MM studies. Methods that directly incorporate fluctuations of electronic properties during dynamics to construct QM regions are currently being developed in our group.

\section{Conflicts of interest}

There are no conflicts to declare.

\section{Acknowledgements}

This work was supported in part by an MIT Research Support Committee NEC Corporation grant. Support for this research was provided by a core center grant P30-ES002109 from the National Institute of Environmental Health Sciences, National Institutes of Health. H. J. K. holds a Career Award at the Scientific Interface from the Burroughs Wellcome Fund, which also supported this work. This work was carried out in part using computational resources from the Extreme Science and Engineering Discovery Environment (XSEDE), which is supported by National Science Foundation grant number ACI-1548562. This work used the XStream computational resource, supported by the National Science Foundation Major Research Instrumentation program (ACI-1429830). The authors thank Adam H. Steeves for providing a critical reading of the manuscript.

\section{References}

1 M. J. Field, P. A. Bash and M. Karplus, A Combined Quantum-Mechanical and Molecular Mechanical Potential for Molecular-Dynamics Simulations, J. Comput. Chem., 1990, 11, 700-733.

2 D. Bakowies and W. Thiel, Hybrid Models for Combined Quantum Mechanical and Molecular Mechanical Approaches, J. Phys. Chem., 1996, 100, 10580-10594.

3 T. Z. Mordasini and W. Thiel, Combined Quantum Mechanical and Molecular Mechanical Approaches, Chimia, 1998, 52, 288-291.

4 G. Monard and K. M. Merz, Combined Quantum Mechanical/ Molecular Mechanical Methodologies Applied to Biomolecular Systems, Acc. Chem. Res., 1999, 32, 904-911.
5 J. Gao and D. G. Truhlar, Quantum Mechanical Methods for Enzyme Kinetics, Annu. Rev. Phys. Chem., 2002, 53, 467-505.

6 E. Rosta, M. Klahn and A. Warshel, Towards Accurate Ab Initio QM/MM Calculations of Free-Energy Profiles of Enzymatic Reactions, J. Phys. Chem. B, 2006, 110, 2934-2941.

7 H. Lin and D. Truhlar, QM/MM: What Have We Learned, Where Are We, and Where Do We Go from Here? Theor. Chem. Acc., 2007, 117, 185-199.

8 A. Warshel and M. Levitt, Theoretical Studies of Enzymic Reactions: Dielectric, Electrostatic and Steric Stabilization of the Carbonium Ion in the Reaction of Lysozyme, J. Mol. Biol., 1976, 103, 227-249.

9 H. M. Senn and W. Thiel, QM/MM Methods for Biomolecular Systems, Angew. Chem., Int. Ed., 2009, 48, 1198-1229.

10 J. Gao, S. Ma, D. T. Major, K. Nam, J. Pu and D. G. Truhlar, Mechanisms and Free Energies of Enzymatic Reactions, Chem. Rev., 2006, 106, 3188-3209.

11 P. Vidossich, G. Florin, M. Alfonso-Prieto, E. Derat, S. Shaik and C. Rovira, On the Role of Water in Peroxidase Catalysis: A Theoretical Investigation of Hrp Compound I Formation, J. Phys. Chem. B, 2010, 114, 5161-5169.

12 P. Carloni, U. Rothlisberger and M. Parrinello, The Role and Perspective of Ab Initio Molecular Dynamics in the Study of Biological Systems, Acc. Chem. Res., 2002, 35, 455-464.

13 K. P. Eurenius, D. C. Chatfield, B. R. Brooks and M. Hodoscek, Enzyme Mechanisms with Hybrid Quantum and Molecular Mechanical Potentials. I. Theoretical Considerations, Int. J. Quantum Chem., 1996, 60, 1189-1200.

14 H. M. Senn and W. Thiel, QM/MM Studies of Enzymes, Curr. Opin. Chem. Biol., 2007, 11, 182-187.

15 A. Monari, J.-L. Rivail and X. Assfeld, Advances in the Local Self-Consistent Field Method for Mixed Quantum Mechanics/Molecular Mechanics Calculations, Acc. Chem. Res., 2012, 46, 596-603.

16 Y. Wang and J. Gao, Projected Hybrid Orbitals: A General QM/MM Method, J. Phys. Chem. B, 2015, 119, 1213-1224.

17 P. Slavicek and T. J. Martinez, Multicentered Valence Electron Effective Potentials: A Solution to the Link Atom Problem for Ground and Excited Electronic States, J. Chem. Phys., 2006, 124, 084107.

18 R. B. Murphy, D. M. Philipp, R. A. Friesner and A. Mixed, Quantum Mechanics/Molecular Mechanics (QM/MM) Method for Large Scale Modeling of Chemistry in Protein Environments, J. Comput. Chem., 2000, 21, 1442-1457.

19 Y. Zhang, T.-S. Lee, W. Yang and A. Pseudobond, Approach to Combining Quantum Mechanical and Molecular Mechanical Methods, J. Chem. Phys., 1999, 110, 46-54.

20 G. A. DiLabio, M. M. Hurley and P. A. Christiansen, Simple One-Electron Quantum Capping Potentials for Use in Hybrid QM/MM Studies of Biological Molecules, J. Chem. Phys., 2002, 116, 9578-9584.

21 O. A. von Lilienfeld, I. Tavernelli, U. Rothlisberger and D. Sebastiani, Variational Optimization of Effective AtomCentered Potentials for Molecular Properties, J. Chem. Phys., 2005, 122, 14113. 
22 B. Wang and D. G. Truhlar, Combined Quantum Mechanical and Molecular Mechanical Methods for Calculating Potential Energy Surfaces: Tuned and Balanced Redistributed Charge Algorithm, J. Chem. Theory Comput., 2010, 6, 359-369.

23 N. M. Thellamurege and H. Hirao, Effect of Protein Environment within Cytochrome P450cam Evaluated Using a Polarizable-Embedding QM/MM Method, J. Phys. Chem. B, 2014, 118, 2084-2092.

24 J. W. Ponder, C. Wu, P. Ren, V. S. Pande, J. D. Chodera, M. J. Schnieders, I. Haque, D. L. Mobley, D. S. Lambrecht and R. A. DiStasio Jr., Current Status of the AMOEBA Polarizable Force Field, J. Phys. Chem. B, 2010, 114, 2549-2564.

25 T. A. Halgren and W. Damm, Polarizable Force Fields, Curr. Opin. Struct. Biol., 2001, 11, 236-242.

26 L. J. Nåbo, J. M. H. Olsen, T. J. Martínez and J. Kongsted, The Quality of the Embedding Potential Is Decisive for Minimal Quantum Region Size in Embedding Calculations: The Case of the Green Fluorescent Protein, J. Chem. Theory Comput., 2017, 13, 6230-6236.

27 A. Ganguly, E. Boulanger and W. Thiel, Importance of Mm Polarization in QM/MM Studies of Enzymatic Reactions: Assessment of the QM/MM Drude Oscillator Model, J. Chem. Theory Comput., 2017, 13, 2954-2961.

28 Q. Li, B. Mennucci, M. A. Robb, L. Blancafort and C. Curutchet, Polarizable QM/MM Multiconfiguration Self-Consistent Field Approach with State-Specific Corrections: Environment Effects on Cytosine Absorption Spectrum, J. Chem. Theory Comput., 2015, 11, 1674-1682.

29 D. Loco, L. Lagardère, S. Caprasecca, F. Lipparini, B. Mennucci and J.-P. Piquemal, Hybrid QM/MM Molecular Dynamics with AMOEBA Polarizable Embedding, J. Chem. Theory Comput., 2017, 13, 4025-4033.

30 I. S. Ufimtsev, N. Luehr and T. J. Martínez, Charge Transfer and Polarization in Solvated Proteins from $A b$ Initio Molecular Dynamics, J. Phys. Chem. Lett., 2011, 2, 1789-1793.

31 G. Nadig, L. C. Van Zant, S. L. Dixon and K. M. Merz, Charge-Transfer Interactions in Macromolecular Systems: A New View of the Protein/Water Interface, J. Am. Chem. Soc., 1998, 120, 5593-5594.

32 H. J. Kulik, N. Luehr, I. S. Ufimtsev and T. J. Martinez, $A b$ Initio Quantum Chemistry for Protein Structure, J. Phys. Chem. B, 2012, 116, 12501-12509.

33 I. S. Ufimtsev and T. J. Martínez, Quantum Chemistry on Graphical Processing Units. 1. Strategies for Two-Electron Integral Evaluation, J. Chem. Theory Comput., 2008, 4, 222-231.

34 I. S. Ufimtsev and T. J. Martínez, Quantum Chemistry on Graphical Processing Units. 2. Direct Self-Consistent-Field Implementation, J. Chem. Theory Comput., 2009, 5, 1004-1015.

35 I. S. Ufimtsev and T. J. Martínez, Quantum Chemistry on Graphical Processing Units. 3. Analytical Energy Gradients, Geometry Optimization, and First Principles Molecular Dynamics, J. Chem. Theory Comput., 2009, 5, 2619-2628.

36 C. M. Isborn, N. Luehr, I. S. Ufimtsev and T. J. Martinez, Excited-State Electronic Structure with Configuration
Interaction Singles and Tamm-Dancoff Time-Dependent Density Functional Theory on Graphical Processing Units, J. Chem. Theory Comput., 2011, 7, 1814-1823.

37 C. Ochsenfeld, J. Kussmann and D. S. Lambrecht, LinearScaling Methods in Quantum Chemistry, Rev. Comput. Chem., 2007, 23, 1.

38 K. Eichkorn, F. Weigend, O. Treutler and R. Ahlrichs, Auxiliary Basis Sets for Main Row Atoms and Transition Metals and Their Use to Approximate Coulomb Potentials, Theor. Chem. Acc., 1997, 97, 119-124.

39 K. Eichkorn, O. Treutler, H. Öhm, M. Häser and R. Ahlrichs, Auxiliary Basis Sets to Approximate Coulomb Potentials, Chem. Phys. Lett., 1995, 240, 283-290.

40 E. Rudberg, E. H. Rubensson and P. Salek, Kohn-Sham Density Functional Theory Electronic Structure Calculations with Linearly Scaling Computational Time and Memory Usage, J. Chem. Theory Comput., 2011, 7, 340-350.

41 M. Challacombe and E. Schwegler, Linear Scaling Computation of the Fock Matrix, J. Chem. Phys., 1997, 106, 5526-5536.

42 C.-K. Skylaris, P. D. Haynes, A. A. Mostofi and M. C. Payne, Introducing ONETEP: Linear Scaling Density Functional Simulations on Parallel Computers, J. Chem. Phys., 2005, $122,084119$.

43 D. R. Bowler and T. Miyazaki, O(N) Methods in Electronic Structure Calculations, Rep. Prog. Phys., 2012, 75, 036503.

$44 \mathrm{~J}$. VandeVondele, U. Borštnik and J. Hutter, Linear Scaling Self-Consistent Field Calculations with Millions of Atoms in the Condensed Phase, J. Chem. Theory Comput., 2012, 8, 3565-3573.

45 G. E. Scuseria, Linear Scaling Density Functional Calculations with Gaussian Orbitals, J. Phys. Chem. A, 1999, 103, 4782-4790.

46 M. Guidon, J. Hutter and J. VandeVondele, Robust Periodic Hartree-Fock Exchange for Large-Scale Simulations Using Gaussian Basis Sets, J. Chem. Theory Comput., 2009, 5, 3010-3021.

47 D. Flaig, M. Beer and C. Ochsenfeld, Convergence of Electronic Structure with the Size of the QM Region: Example of QM/MM NMR Shieldings, J. Chem. Theory Comput., 2012, 8, 2260-2271.

48 J. D. Hartman, T. J. Neubauer, B. G. Caulkins, L. J. Mueller and G. J. Beran, Converging Nuclear Magnetic Shielding Calculations with Respect to Basis and System Size in Protein Systems, J. Biomol. NMR, 2015, 62, 327-340.

49 S. J. Fox, C. Pittock, T. Fox, C. S. Tautermann, N. Malcolm and C. K. Skylaris, Electrostatic Embedding in Large-Scale First Principles Quantum Mechanical Calculations on Biomolecules, J. Chem. Phys., 2011, 135, 224107.

50 R. Z. Liao and W. Thiel, Convergence in the QM-Only and QM/MM Modeling of Enzymatic Reactions: A Case Study for Acetylene Hydratase, J. Comput. Chem., 2013, 34, 2389-2397.

51 K. Sadeghian, D. Flaig, I. D. Blank, S. Schneider, R. Strasser, D. Stathis, M. Winnacker, T. Carell and C. Ochsenfeld, Ribose-Protonated DNA Base Excision Repair: A Combined Theoretical and Experimental Study, Angew. Chem., Int. Ed., 2014, 53, 10044-10048. 
52 H. J. Kulik, J. Zhang, J. P. Klinman and T. J. Martinez, How Large Should the QM Region Be in QM/MM Calculations? The Case of Catechol O-Methyltransferase, J. Phys. Chem. B, 2016, 120, 11381-11394.

53 I. Solt, P. Kulhanek, I. Simon, S. Winfield, M. C. Payne, G. Csanyi and M. Fuxreiter, Evaluating Boundary Dependent Errors in QM/MM Simulations, J. Phys. Chem. B, 2009, 113, 5728-5735.

54 C. M. Isborn, A. W. Goetz, M. A. Clark, R. C. Walker and T. J. Martinez, Electronic Absorption Spectra from $\mathrm{Mm}$ and $A b$ Initio QM/MM Molecular Dynamics: Environmental Effects on the Absorption Spectrum of Photoactive Yellow Protein, J. Chem. Theory Comput., 2012, 8, 5092-5106.

55 D. E. Vanpoucke, J. Oláh, F. De Proft, V. Van Speybroeck and G. Roos, Convergence of Atomic Charges with the Size of the Enzymatic Environment, J. Chem. Inf. Model., 2015, 55, 564-571.

56 T. V. Harris and R. K. Szilagyi, Protein Environmental Effects on Iron-Sulfur Clusters: A Set of Rules for Constructing Computational Models for Inner and Outer Coordination Spheres, J. Comput. Chem., 2016, 37, 1681-1696.

57 L. Hu, P. r. Söderhjelm and U. Ryde, On the Convergence of QM/MM Energies, J. Chem. Theory Comput., 2011, 7, 761-777.

58 S. Roßbach and C. Ochsenfeld, Influence of Coupling and Embedding Schemes on QM Size Convergence in QM/MM Approaches for the Example of a Proton Transfer in DNA, J. Chem. Theory Comput., 2017, 13, 1102-1107.

59 M. R. Provorse, T. Peev, C. Xiong and C. M. Isborn, Convergence of Excitation Energies in Mixed Quantum and Classical Solvent: Comparison of Continuum and Point Charge Models, J. Phys. Chem. B, 2016, 120, 12148-12159.

$60 \mathrm{~J}$. M. Milanese, M. R. Provorse, E. Alameda and C. M. Isborn, Convergence of Computed Aqueous Absorption Spectra with Explicit Quantum Mechanical Solvent, J. Chem. Theory Comput., 2017, 13, 2159-2171.

61 A. Morgenstern, M. Jaszai, M. E. Eberhart and A. N. Alexandrova, Quantified Electrostatic Preorganization in Enzymes Using the Geometry of the Electron Charge Density, Chem. Sci., 2017, 8, 5010-5018.

62 R.-Z. Liao and W. Thiel, Comparison of QM-only and QM/ MM Models for the Mechanism of Tungsten-Dependent Acetylene Hydratase, J. Chem. Theory Comput., 2012, 8, 3793-3803.

63 P. A. Bash, M. J. Field, R. C. Davenport, G. A. Petsko, D. Ringe and M. Karplus, Computer Simulation and Analysis of the Reaction Pathway of Triosephosphate Isomerase, Biochemistry, 1991, 30, 5826-5832.

64 L. Hu, J. Eliasson, J. Heimdal and U. Ryde, Do Quantum Mechanical Energies Calculated for Small Models of Protein-Active Sites Converge? J. Phys. Chem. A, 2009, 113, 11793-11800.

65 L. Hu, P. Söderhjelm and U. Ryde, Accurate Reaction Energies in Proteins Obtained by Combining QM/MM and Large QM Calculations, J. Chem. Theory Comput., 2012, 9, 640-649.
66 S. Sumner, P. Söderhjelm and U. Ryde, Effect of Geometry Optimizations on QM-Cluster and QM/MM Studies of Reaction Energies in Proteins, J. Chem. Theory Comput., 2013, 9, 4205-4214.

67 M. Karelina and H. J. Kulik, Systematic Quantum Mechanical Region Determination in QM/MM Simulation, J. Chem. Theory Comput., 2017, 13, 563-576.

68 H. W. Qi, M. Karelina and H. J. Kulik, Quantifying Electronic Effects in QM and QM/MM Biomolecular Modeling with the Fukui Function, Acta Phys.-Chim. Sin., 2018, 34, 81-91.

69 K. Meier, W. Thiel and W. F. van Gunsteren, On the Effect of a Variation of the Force Field, Spatial Boundary Condition and Size of the QM Region in QM/MM MD Simulations, J. Comput. Chem., 2012, 33, 363-378.

70 N. Patra, E. I. Ioannidis and H. J. Kulik, Computational Investigation of the Interplay of Substrate Positioning and Reactivity in Catechol O-Methyltransferase, PLoS One, 2016, 11, e0161868.

71 J. Lameira, R. P. Bora, Z. T. Chu and A. Warshel, Methyltransferases Do Not Work by Compression, Cratic, or Desolvation Effects, but by Electrostatic Preorganization, Proteins: Struct., Funct., Bioinf., 2015, 83, 318-330.

72 G. Jindal and A. Warshel, Exploring the Dependence of QM/MM Calculations of Enzyme Catalysis on the Size of the QM Region, J. Phys. Chem. B, 2016, 120, 9913-9921.

73 M. A. Olsson and U. Ryde, Comparison of QM/MM Methods to Obtain Ligand-Binding Free Energies, J. Chem. Theory Comput., 2017, 13, 2245-2253.

74 J. Řezáč and P. Hobza, Advanced Corrections of Hydrogen Bonding and Dispersion for Semiempirical Quantum Mechanical Methods, J. Chem. Theory Comput., 2011, 8, 141-151.

$75 \mathrm{~J}$. Axelrod and R. Tomchick, Enzymatic O-Methylation of Epinephrine and Other Catechols, J. Biol. Chem., 1958, 233, 702-705.

76 A. Razin and A. D. Riggs, DNA Methylation and Gene Function, Science, 1980, 210, 604-610.

77 M. M. Skinner, J. M. Puvathingal, R. L. Walter and A. M. Friedman, Crystal Structure of Protein Isoaspartyl Methyltransferase: A Catalyst for Protein Repair, Structure, 2000, 8, 1189-1201.

78 J. E. Visick, H. Cai and S. Clarke, The L-Isoaspartyl Protein Repair Methyltransferase Enhances Survival of Aging Escherichia Coli Subjected to Secondary Environmental Stresses, J. Bacteriol., 1998, 180, 2623-2629.

79 R. Salomon-Ferrer, D. A. Case and R. C. Walker, An Overview of the Amber Biomolecular Simulation Package, Wiley Interdiscip. Rev.: Comput. Mol. Sci., 2013, 3, 198-210.

80 D. A. Case, J. T. Berryman, R. M. Betz, D. S. Cerutti, T. E. Cheatham, III, T. A. Darden, R. E. Duke, T. J. Giese, H. Gohlke, A. W. Goetz, N. Homeyer, S. Izadi, P. Janowski, J. Kaus, A. Kovalenko, T. S. Lee, S. LeGrand, P. Li, T. Luchko, R. Luo, B. Madej, K. M. Merz, G. Monard, P. Needham, H. Nguyen, H. T. Nguyen, I. Omelyan, A. Onufriev, D. R. Roe, A. Roitberg, R. Salomon-Ferrer, 
C. L. Simmerling, W. Smith, J. Swails, R. C. Walker, J. Wang, R. M. Wolf, X. Wu, D. M. York and P. A. Kollman, Amber 2015, University of California, San Francisco, 2015.

81 Petachem, http://www.petachem.com, (accessed January 1, 2018).

82 M. A. Rohrdanz, K. M. Martins, J. M. Herbert and A. LongRange-Corrected Density, Functional That Performs Well for Both Ground-State Properties and Time-Dependent Density Functional Theory Excitation Energies, Including Charge-Transfer Excited States, J. Chem. Phys., 2009, 130, 054112.

83 R. Ditchfield, W. J. Hehre and J. A. Pople, Self-Consistent Molecular Orbital Methods. 9. Extended Gaussian-Type Basis for Molecular Orbital Studies of Organic Molecules, J. Chem. Phys., 1971, 54, 724.

84 S. Grimme, J. Antony, S. Ehrlich, H. Krieg and A. Consistent, and Accurate $A b$ Initio Parametrization of Density Functional Dispersion Correction (DFT-D) for the 94 Elements H-Pu, J. Chem. Phys., 2010, 132, 154104.

85 V. Hornak, R. Abel, A. Okur, B. Strockbine, A. Roitberg and C. Simmerling, Comparison of Multiple Amber Force Fields and Development of Improved Protein Backbone Parameters, Proteins: Struct., Funct., Bioinf., 2006, 65, 712-725.

86 J. A. Maier, C. Martinez, K. Kasavajhala, L. Wickstrom, K. E. Hauser and C. Simmerling, Ff14sb: Improving the Accuracy of Protein Side Chain and Backbone Parameters from Ff99sb, J. Chem. Theory Comput., 2015, 11, 3696-3713.

87 W. L. Jorgensen, J. Chandrasekhar, J. D. Madura, R. W. Impey and M. L. Klein, Comparison of Simple Potential Functions for Simulating Liquid Water, J. Chem. Phys., 1983, 79, 926-935.

88 K. Rutherford, I. Le Trong, R. E. Stenkamp and W. W. Parson, Crystal Structures of Human $108 \mathrm{v}$ and $108 \mathrm{~m}$ Catechol O-Methyltransferase, J. Mol. Biol., 2008, 380, 120-130.

89 M. Souaille and B. Roux, Extension to the Weighted Histogram Analysis Method: Combining Umbrella Sampling with Free Energy Calculations, Comput. Phys. Commun., 2001, 135, 40-57.

90 S. Kumar, J. M. Rosenberg, D. Bouzida, R. H. Swendsen and P. A. Kollman, The Weighted Histogram Analysis Method for Free-Energy Calculations on Biomolecules. I. The Method, J. Comput. Chem., 1992, 13, 1011-1021.

91 A. Grossfield, "Wham: The Weighted Histogram Analysis Method”, Version 2.0.9, http://membrane.urmc.rochester. edu/content/wham, (accessed January 1, 2018).

92 G. M. Torrie and J. P. Valleau, Nonphysical Sampling Distributions in Monte Carlo Free-Energy Estimation: Umbrella Sampling, J. Comput. Phys., 1977, 23, 187-199.

93 T. Lotta, J. Vidgren, C. Tilgmann, I. Ulmanen, K. Melen, I. Julkunen and J. Taskinen, Kinetics of Human Soluble and Membrane-Bound Catechol $O$-Methyltransferase: A Revised Mechanism and Description of the Thermolabile Variant of the Enzyme, Biochemistry, 1995, 34, 4202-4210.

94 M. F. Hegazi, R. T. Borchardt and R. L. Schowen, Alpha.-Deuterium and Carbon-13 Isotope Effects for Methyl Transfer Catalyzed by Catechol $O$-Methyltransferase.
Sn2-Like Transition State, J. Am. Chem. Soc., 1979, 101, 4359-4365.

95 R. W. Woodard, M. D. Tsai, H. G. Floss, P. A. Crooks and J. K. Coward, Stereochemical Course of the Transmethylation Catalyzed by Catechol O-Methyltransferase, J. Biol. Chem., 1980, 255, 9124-9127.

96 J. Axelrod, J. Methylation Reactions in the Formation and Metabolism of Catecholamines and Other Biogenic Amines, Pharmacol. Rev., 1966, 18, 95-113.

97 J. K. Coward, E. P. Slisz and F. Y. H. Wu, Kinetic Studies on Catechol $O$-Methyltransferase, Product Inhibition and the Nature of the Catechol Binding Site, Biochemistry, 1973, 12, 2291-2297.

98 J. Vidgren, L. A. Svensson and A. Liljas, Crystal Structure of Catechol O-Methyltransferase, Nature, 1994, 368, 354-358.

99 E. Schultz and E. Nissinen, Inhibition of Rat Liver and Duodenum Soluble Catechol-O-Methyltransferase by a Tight-Binding Inhibitor or-462, Biochem. Pharmacol., 1989, 38, 3953-3956.

100 P. Lautala, I. Ulmanen and J. Taskinen, Molecular Mechanisms Controlling the Rate and Specificity of Catechol $O$-Methylation by Human Soluble Catechol O-Methyltransferase, Mol. Pharmacol., 2001, 59, 393-402.

101 J. Zhang and J. P. Klinman, Enzymatic Methyl Transfer: Role of an Active Site Residue in Generating Active Site Compaction That Correlates with Catalytic Efficiency, J. Am. Chem. Soc., 2011, 133, 17134-17137.

102 T. H. Rod and U. Ryde, Quantum Mechanical Free Energy Barrier for an Enzymatic Reaction, Phys. Rev. Lett., 2005, 94, 138302.

103 T. H. Rod and U. Ryde, Accurate QM/MM Free Energy Calculations of Enzyme Reactions: Methylation by Catechol O-Methyltransferase, J. Chem. Theory Comput., 2005, 1, 1240-1251.

104 M. Roca, V. Moliner, I. Tuñón and J. T. Hynes, Coupling between Protein and Reaction Dynamics in Enzymatic Processes: Application of Grote-Hynes Theory to Catechol O-Methyltransferase, J. Am. Chem. Soc., 2006, 128, 6186-6193.

105 G. D. Ruggiero, I. H. Williams, M. Roca, V. Moliner and I. Tuñón, QM/MM Determination of Kinetic Isotope Effects for COMT-Catalyzed Methyl Transfer Does Not Support Compression Hypothesis, J. Am. Chem. Soc., 2004, 126, 8634-8635.

106 B. Kuhn and P. A. Kollman, QM-Fe and Molecular Dynamics Calculations on Catechol $O$-Methyltransferase: Free Energy of Activation in the Enzyme and in Aqueous Solution and Regioselectivity of the Enzyme-Catalyzed Reaction, J. Am. Chem. Soc., 2000, 122, 2586-2596.

107 M. Roca, V. Moliner, J. J. Ruiz-Pernía, E. Silla and I. Tuñón, Activation Free Energy of Catechol $O$-Methyltransferase. Corrections to the Potential of Mean Force, J. Phys. Chem. A, 2006, 110, 503-509.

108 J. J. P. Stewart, Optimization of Parameters for Semiempirical Methods V: Modification of NDDO Approximations and Application to 70 Elements, J. Mol. Model., 2007, 13, 1173-1213. 
109 N. Kanaan, J. J. Ruiz Pernia and I. H. Williams, QM/MM Simulations for Methyl Transfer in Solution and Catalysed by COMT: Ensemble-Averaging of Kinetic Isotope Effects, Chem. Commun., 2008, 6114-6116.

110 M. Marianski, A. Supady, T. Ingram, M. Schneider and C. Baldauf, Assessing the Accuracy of across-the-Scale Methods for Predicting Carbohydrate Conformational Energies for the Examples of Glucose and A-Maltose, J. Chem. Theory Comput., 2016, 12, 6157-6168.

111 P. Zhang, P. Bao and J. Gao, Dipole Preserving and Polarization Consistent Charges, J. Comput. Chem., 2011, 32, 2127-2139.

112 P. Jurečka, J. Šponer, J. Černý and P. Hobza, Benchmark Database of Accurate (MP2 and CCSD (T) Complete Basis Set Limit) Interaction Energies of Small Model Complexes, DNA Base Pairs, and Amino Acid Pairs, Phys. Chem. Chem. Phys., 2006, 8, 1985-1993.
113 C. M. Isborn, B. D. Mar, B. F. Curchod, I. Tavernelli and T. J. Martinez, The Charge Transfer Problem in Density Functional Theory Calculations of Aqueously Solvated Molecules, J. Phys. Chem. B, 2013, 117, 12189-12201.

114 J. Zhang, H. J. Kulik, T. J. Martinez and J. P. Klinman, Mediation of Donor-Acceptor Distance in an Enzymatic Methyl Transfer Reaction, Proc. Natl. Acad. Sci. U. S. A., 2015, 112, 7954-7959.

115 D. Rutkowska-Zbik, M. Witko and L. Fiedor, Ligation of Water to Magnesium Chelates of Biological Importance, J. Mol. Model., 2013, 19, 4661-4667.

116 W. W. Cleland, P. A. Frey and J. A. Gerlt, The Low Barrier Hydrogen Bond in Enzymatic Catalysis, J. Biol. Chem., 1998, 273, 25529-25532.

117 U. Ryde, How Many Conformations Need to Be Sampled to Obtain Converged QM/MM Energies? The Curse of Exponential Averaging, J. Chem. Theory Comput., 2017, 13, 5745-5752. 